\title{
Mechanical Examination of Chicken Vessel with Custom- Built Experimental Equipment
}

\author{
Ádám MEISEL, ${ }^{1} \mathrm{~K}$. Brigitta TÓTH, ${ }^{2}$ Éva LAKATOS ${ }^{3}$ \\ ${ }^{1}$ Budapest University of Technology and Economics, Department of Materials Science and Engineering, \\ Budapest, Hungary, ameisel@edu.bme.hu \\ ${ }^{2}$ Budapest University of Technology and Economics, Faculty of Civil Engineering, Departament of struc- \\ tural Mechanics, Budapest, Hungary, brigitta.toth@mail.bme.hu \\ ${ }^{3}$ Budapest University of Technology and Economics, Faculty of Civil Engineering, Departament of struc- \\ tural Mechanics, Hungary, lakatos.eva@emk.bme.hu
}

\begin{abstract}
Cardiovascular disease has been decimating humanity for decades. In vivo examination of blood vessels is of great help in the development of numerical models and simulations that can help physicians significantly improve sufferers' quality of life. For such models, the different mechanical characteristics of the vessels are the input data. Several such mechanical properties of the vessels, such as modulus of elasticity and tensile strength, are determined by a tensile test. In the course of our research, an experimental device was developed and tested which is suitable for biaxial tensile tests of blood vessels, which we present through the examination of chicken blood vessels.
\end{abstract}

Keywords: biaxial tensile testing, mechanical properties, experimental device.

\section{Introduction}

Cardiovascular diseases have been a leading cause of death for decades. The WHO and several organizations have already identified the risk factors leading to the disease and the possibilities of prevention. Nevertheless, there has been very little improvement in the number of deaths from the disease over the years [1, 2]. Because of all this, the development of devices to treat such diseases is reaching its heyday. Such devices (stent, flow diverter stent, WEB, etc.) are most often inserted into the vessel by the physician. Mapping the different properties of the vessel wall is also essential for the development of tools and a deeper understanding of the problem area. Because most of these problematic vascular sections or in vivo studies of the implant are difficult, researchers are able to draw conclusions based on input data from in vitro measurements using various material and mechanical models and simulations [3-6].

There are results from mechanical examination of intracranial aneurysms, but most of these perform only uniaxial examination and do not provide any data on vascular clamping methods, which are significant for proper results [7-9].

Vascular wall samples obtained during surgery or from the dead are usually placed immediately or after a short freezing period in $37.5{ }^{\circ} \mathrm{C}$ saline at pH $7.4 \mathrm{pH}$, which is adequately saturated with oxygen, and the tensile test is performed [10].

Our goal is to further develop a device with which it is possible to determine the mechanical properties of the vessels along two axes in a reliable manner, even without the destruction of the vessels prior to testing.

\section{Materials and methods}

\subsection{Experimental device}

In our research, we used a custom-designed biaxial tensile machine (Figure 1). The sample is fixed at four points with a clamp. Each clamp is connected directly to the arms or load cells. The arms are connected to carriages running on linear wires, which are moved by means of four stepper motors and a threaded spindle. 
An important aspect during the measurements was that the properties of the study environment were as close as possible to the human body. The saline solution at the appropriate temperature and $\mathrm{pH}$ is placed in a tub that can be raised, thereby facilitating the capture process.

\subsection{Clamp design and manufacture}

From the point of view of the tensile test, the decisive point outside the device is the appropriate clamping design. In our case, this plays a prominent role, as the examined samples are very thin, easily torn and damaged. Consequently, the most important requirement for a clamp is to clamp and hold the sample intact during testing. There must be no stress collection point on the sample in the clamping environment, as this may result in erroneous results.

We designed and evaluated several concepts, the most promising of which were manufactured and tested. Such was the case with the "rake" design or the spring-loaded clamp. The most optimal design proved to be a screw-tensioned clamp in which the sample is clamped between teeth, in front of which there is a rounded strain relief part. We first tried a geometry with a pointed tip that is equally suitable for capturing smaller and larger samples. However, the measurements revealed that rupture was most likely to start next to the slider, so we finally left the tip and made the end of the chuck straight. The clamps were manufactured using a Creality Ender 3 type 3D printer (Figure 2).

\subsection{Measuring method}

The force-displacement curve characteristic of the sample can be determined with the help of the tensile device. The force is measured on the two axes with a load cell. The displacement is calculated from the number of steps of the stepping motors.

The measurement is started by fixing the sample. First, the sample is placed on the lower clamping half, and then the upper clamping half is tightened by a screw. During our measurements, low-density polyethylene (LDPE) film and chicken neck and abdominal vessels were examined. The LDPE sample was cut according to ASTM D638 Type V with a broken surface size of $9.53 \mathrm{~mm} \times 3.18 \mathrm{~mm}$. Using a unique template, $14 \mathrm{~mm} \times 2.5 \mathrm{~mm}$ ruptured surface samples were prepared from the vessels. The test was performed in each case at $20^{\circ} \mathrm{C}$, the measurement speed was $5 \mathrm{~mm} / \mathrm{min}$ and the sampling frequency was $16.7 \mathrm{~Hz}$. The vessels

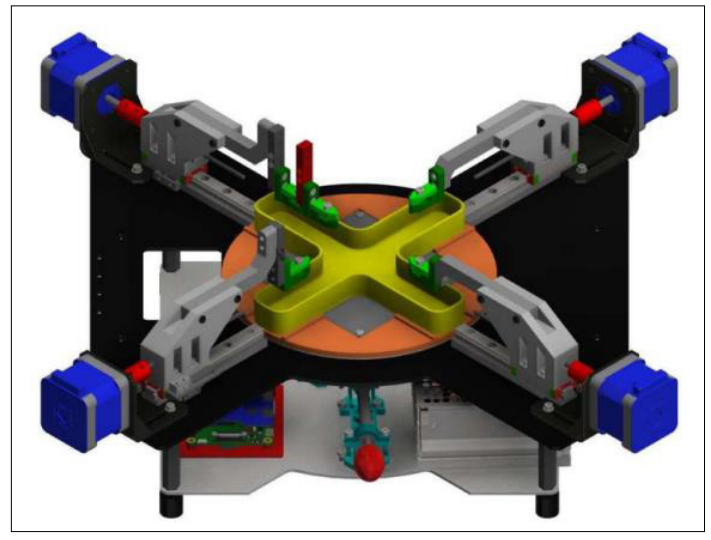

Figure 1. Design of the experimental equipment.

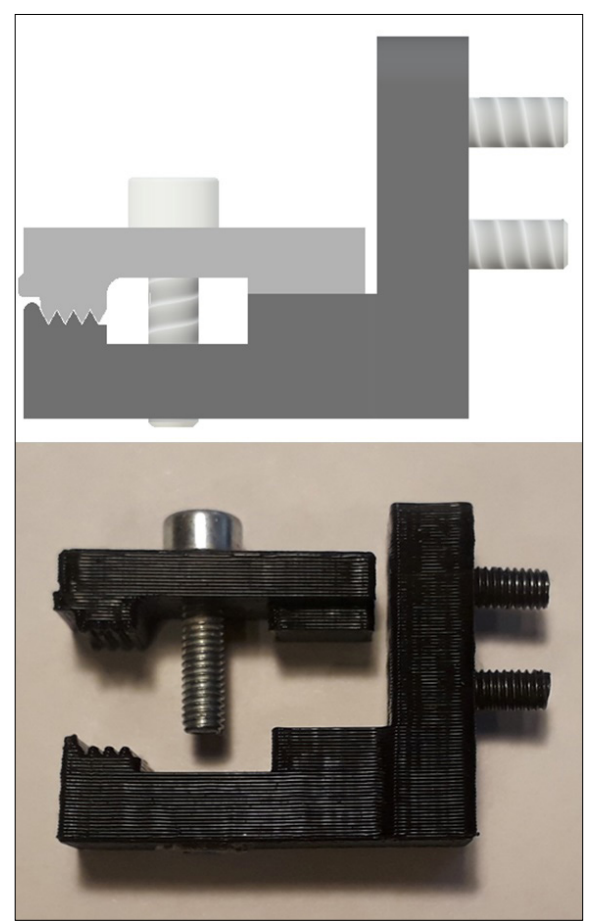

Figure 2. Clamp design, top: model, bottom: additively made clamp.

were removed from the $0.9 \%$ physiological saline immediately before measurement.

\section{Results}

During the tensile test, it was clearly established that the vessels were not torn at the clamp, so the design of the clamp was appropriate (Figure 3).

The curve shown in Figure 4 was obtained during the study. This shows the tensile curve drawn by the X and Y axes of the apparatus for LDPE foil and chicken vessel specimens. 


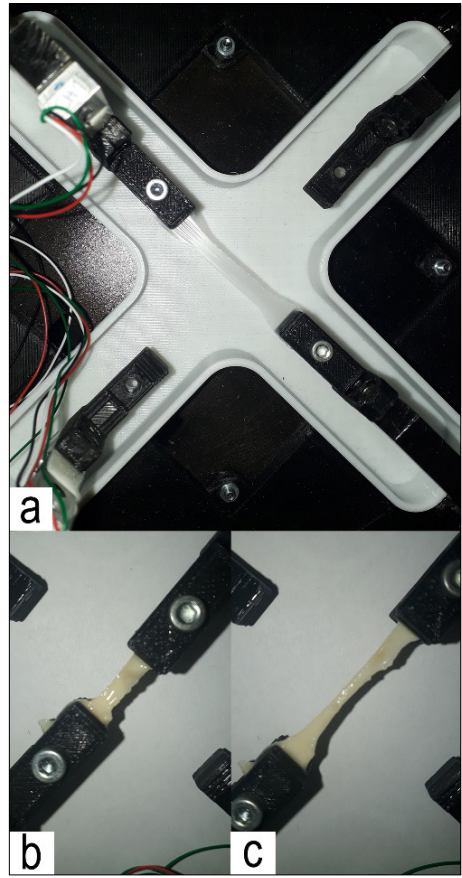

Figure 3. Clamp testing on a) LDPE foil specimen and chicken carotid artery piece, $b$ ) vessel at pull, c) vessel at rupture.

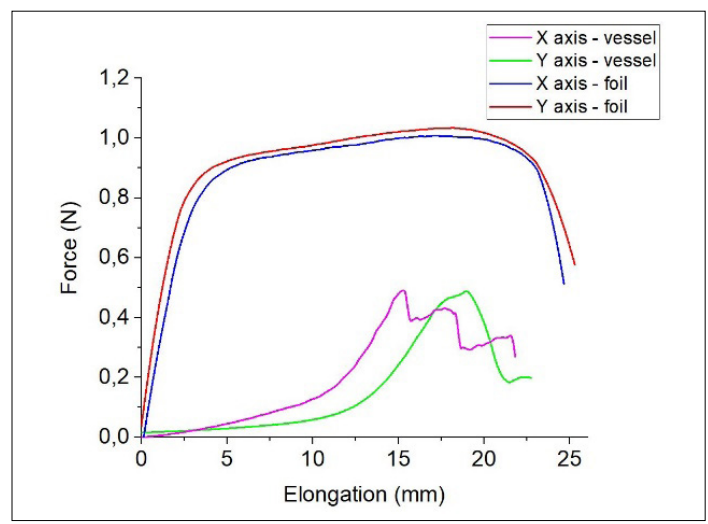

Figure 4. Tensile curves during examination of LDPE film specimens and chicken vessels.

\section{Conclusions}

The clamp we design and manufacture can be suitable for tearing thin tissues (blood vessels, even tendons, ligaments, etc.) without damaging the tissue. The obtained values correspond to the results of the literature [8] in shape and order of magnitude, so the measuring device is suitable for the tensile testing of blood vessels.

The next step in our research is to validate the equipment using a tensile machine with a simi- larly small measuring cell, and then to perform a single- and biaxial tensile test on human vessels.

\section{Acknowledgments}

The publication of the work reported herein has been supported by the NTP-SZKOLL-20-0067 National Talent Programme of the Ministry of Human Capacities and Higher Education Excellence Program of the Ministry of Human Capacities in the frame of Biotechnology research area of Budapest University of Technology and Economics (BME FIKP-BIO). The research reported in this paper and carried out at BME has been supported by the NRDI Fund (TKP2020 IES, Grant No. BME-IE-NAT; TKP2020 NC, Grant No. BME-NCS) based on the charter of bolster issued by the NRDI Office under the auspices of the Ministry for Innovation and Technology.

\section{References}

[1] Ard J. D., Franklin F. A.: Cardiovascular Disease. Handbook of Clinical Nutrition ( $4^{\text {th }}$ Edition) 2006, 422-447.

https://doi.org/10.1016/B978-0-323-03952-9.50026-X

[2] WHO: 2008-2013 Action Plan for the Global Strategy for the Prevention and Control of Non-communicable Diseases: Prevent and Control Cardiovascular Diseases, Cancers, Chronic Respiratory Diseases and Diabetes. 2009.

www.who.int/publications/i/item/9789241597418

[3] Szabó V., Jenei C., Halász G.: Modelling Blood Pressure in Stenosed Coronary Arteries. Periodica Polytechnica Mechanical Engineering, 61/3. (2017) 242-246.

https://doi.org/10.3311/PPme.10469

[4] Károly D., Asztalos L., Micsík T., Szabó PJ.: Non-Destructive Analysis of Explanted Coronary Artery Stents. Acta Polytechnica Hungarica 14/2. (2017) 171-181.

https://doi.org/10.12700\%2FAPH.14.2.2017.2.9

[5] Tóth B. K., Bojtár I.: Validation of the Hyperelastic Material Parameters of Healthy Human Brain Arteries and Cerebral Saccular Aneurysms. $5^{\text {th }}$ European Conference of the International Federation for Medical and Biological Engineering, 876-879. https://doi.org/10.1007/978-3-642-23508-5_228

[6] Asztalos L., Károly D.: Examination of pathological stents. Müszaki tudományos közlemények 5. (2016) 73-76.

https://doi.org/10.33895/mtk-2016.05.09

[7] Lally C., Reid A. J., Prendergast P. J.: Elastic Behavior of Porcine Coronary Artery Tissue Under Uniaxial and Equibiaxial Tension. Annals of Biomedical Engineering 32 (2004), 1355-1364. https://doi.org/10.1114/B:ABME.0000042224.23927.ce

[8] Costalat V., Sanchez M., Ambard D., Thines L., Lonjon N., Nicoud F., Jourdan F.: Biomechanical Wall Properties of Human Intracranial An- 
eurysms Resected Following Surgical Clipping (IRRAs Project). Journal of Biomechanics. 44/15. (2011) 2685-2691.

https://doi.org/10.1016/j.jbiomech.2011.07.026

[9] Kural M. H., Cai M., Tang D., Gwyther T., Zheng J., Billiar K. L.: Planar Biaxial Characterization of Diseased Human Coronary and Carotid Arteries for Computational Modeling. Journal of Biome- chanics. 45/5. (2012) 790-798.

https://doi.org/10.1016/j.jbiomech.2011.11.019

[10] Tóth B. K., Nasztanovics F., Bojtár I.: Laboratory Tests for Strength Paramaters of Brain Aneurysms. Acta of Bioengineering and Biomechanics. 9/2. (2007) 3-7.

https://www.actabio.pwr.wroc.pl/Vol9No2/1.pdf 